\title{
Lossless Watermarking Algorithm Based on Multiple-Base Notational System and Pixel Difference
}

\author{
Huan Song ${ }^{1 *}$, Jiatao Kang ${ }^{2}$, Zaihui Cao ${ }^{3}$ \\ ${ }^{1}$ Department of Information Technology, henan polytechnic, Ping'an Street, Zhengzhou, China \\ ${ }^{2}$ Department of Architectural Engineering, Henan College of Transportation School, Tonghui Street, \\ zhengzhou, China \\ ${ }^{3}$ Department of Art and Design, Zhengzhou University of Aeronautics, wenyuan Street, Zhengzhou, \\ China \\ *Corresponding author.
}

\begin{abstract}
In order to identify the authenticity of the industrial packaging, the solution to the current watermark technology is mainly to embed the same watermark capacity into each pixel value in the image, so that the watermark capacity is small, and the anti-distortion performance is not good. Data watermarking algorithm based on system and pixel difference. First divide the carrier image into non-overlapping sub-blocks, and calculate the pixel difference of each sub-block; introduce a hybrid system to obtain different bases. According to the pixel difference, the base selection rule is defined; considering the human visual characteristics, the pixel interval adaptive adjustment mechanism is designed to improve the coding technology and complete the watermark information embedding; finally, the watermark information extraction mechanism is established to restore the watermark. The experimental results show that compared with the current data watermarking algorithm, the proposed algorithm has ideal imperceptibility, as well as larger watermark capacity and lower distortion.
\end{abstract}

Keywords: Industrial industry, Image watermarking, Multiple-base notational system, Pixel difference; Base selection rule, Adaptive adjustment mechanism of pixel interval, Diamond encoding

\section{Introduction}

Digital images have important application value in the fields of economy and medicine because they contain hidden information of many users and have ideal visual expression ability [1-2]. In order to ensure that user data can resist external attacks in the network, scholars have proposed corresponding image watermarking technology [3-4]. For example, Zhang et al. [5] proposed a watermarking technique based on integer wavelet transform and bit level set. The experimental results show that the watermarking scheme has high imperceptibility and robustness. Chen et al. [6] designed a robust watermarking technique based on salient image features. The salient features were used as reference points, and the key data was embedded in it to obtain a complete watermark image. The experimental results verified the rationality and excellence of the watermarking technique. Sex. Xiong Xiangguang et al. [7] presented a robust adaptive watermarking project based on LWT-SVD. Firstly, the watermark information is encrypted, and the original carrier image is not overlapped, and the watermark data embedding mechanism is designed. The key data is embed in the sub-block, and the experimental results indicate that the watermark project has strong robustness.

Although such a watermarking algorithm can better embed user key data into a carrier image and has high imperceptibility and robustness, it can ensure images are safely transmitted in the network; however, these watermarking technologies are essentially data. The equalization embedding mechanism does not consider the characteristics of each pixel in the carrier image, and embeds watermark data of equal capacity into each pixel, so that the extracted watermark has large distortion, and the capacity of the watermark algorithm is small.

ISSN: 0010-8189

(C) CONVERTER 2020

www.converter-magazine.info 
In order to reduce the distortion of the recovery carrier and improve its watermark capacity, this paper proposes a data watermarking algorithm for the hybrid system and pixel difference. The carrier image is segmented into nonoverlapping sub-blocks, and the difference between every two pixel values in the sub-block is computed. At the same time, the watermark data to be embedded is converted into a decimal integer by a mixed cryptosystem to obtain different cardinalities. The cardinality selection rule is defined by using the pixel difference of the sub-block. And the analysis and research of diamond coding technology, the human visual characteristics are introduced into it, and the two pixel adaptive adjustment mechanism is designed to improve it, and the larger watermark data is embedded into the sub-block pixel pair with larger base number. The embedding of watermark information improves the watermark capacity and reduces the distortion of the algorithm. Finally, the information density and watermark capacity of the proposed watermarking algorithm are verified.

\section{Diamond Coding Technology}

The diamond coding technique [8] mainly uses two pixels (pi, 1,pi, 2) as embedding units, embedding the watermark data into the corresponding matrix $(B=2 k 2+2 k+1) B$, and $k \geqslant 1$ is the embedding parameter. According to the diamond coding technique [8], in order to embed the watermark data into the carrier, the minimum embedding parameter $\mathrm{k}$ is determined according to the following function:

$$
\left\lfloor\frac{M_{1} \times M_{2}}{2} \log _{2}\left(2 k^{2}+2 k+1\right)\right\rfloor \geq|S|
$$

Where $|S|$ is the length of the watermark data $S$; M1 and M2 are the number of rows and columns of the carrier image, respectively.

The embedded parameter $k$ is determined in accordance with (1), the set $\Psi\left(p_{i, 1}, p_{i, 2}\right)$ consisting of the carrier image pixels is:

$$
\Psi\left(p_{i, 1}, p_{i, 2}\right)=\left\{(a, b)|| a-p_{i, 1}|+| b-p_{i, 2} \mid \leq k\right\}
$$

According to equation (2), the diamond eigenvalue $D$ of every element $(a, b)$ in the set $\Psi\left(p_{i, 1}, p_{i, 2}\right)$ can be calculated by the following function:

$$
f(a, b)=\bmod ((2 k+1) a+b, B)
$$

In order to conceal the watermark information $S_{B}$ in the $\mathrm{B}$ matrix into the pixel pair $\left(p_{i, 1}, p_{i, 2}\right)$, a coordinate $\left(p_{i, 1}^{\prime}, p_{i, 2}^{\prime}\right)$ is determined according to $D$ of the formula (2) to contented:

$$
f\left(p_{i, 1}^{\prime}, p_{i, 2}^{\prime}\right)=S_{B}
$$

In the watermark data embedding process, in order to prevent the gray value of some pixels $p^{\prime}{ }_{x}$ from exceeding the limit of $[0,255]$, the diamond coding technique overcomes this problem by modifying $p_{x}^{\prime}{ }_{x}$ to $p^{\prime \prime}{ }_{x}$ :

$$
p^{\prime \prime}{ }_{x}= \begin{cases}p^{\prime}{ }_{x}-B & p^{\prime}{ }_{x}>255 \\ p^{\prime}{ }_{x}+B & p^{\prime}{ }_{x}<0\end{cases}
$$

In order to elaborate the diamond coding technique, this paper describes the embedded parameter $k=3$. Assuming that the pixel pair $\Psi(11,19)$ is the embedding target of the watermark data $1_{25}$ to which the base 25 corresponds, the set of sub-pixel pairs $\Psi(11,19)$ is shown in FIG. Since $f(12,17)=1$ in $\Psi(11,19)$, the embedding of the watermark information is realized by replacing $(11,19)$ with the pixel $(12,17)$. Meanwhile, in order to extract the watermark data, $f(12,17)=1$ is obtained by calculating the diamond feature value $D$ of $(12,17)$, and therefore, the corresponding embedded data is $1_{25}$.

ISSN: 0010-8189

(C) CONVERTER 2020 


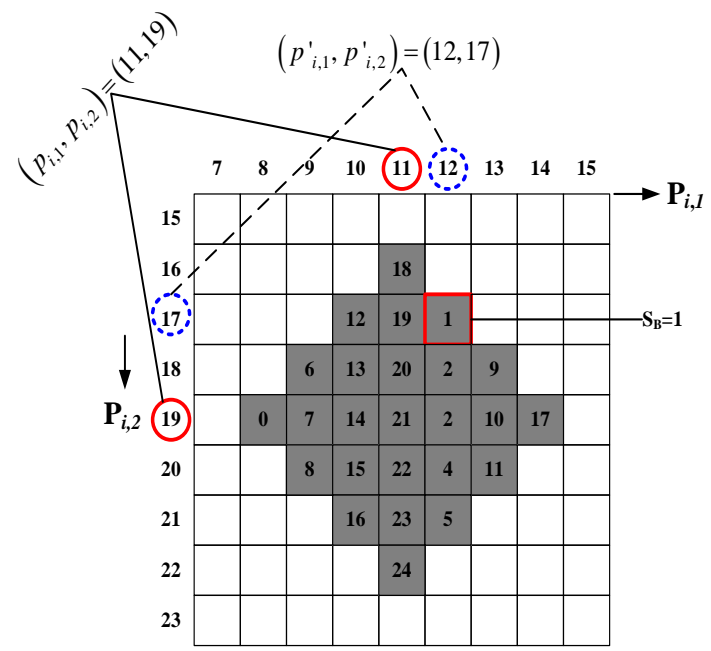

Fig. 1: Pixel set $\Psi(11,19)$ and its corresponding eigenvalues $D$

Although the watermarking technology based on Diamond coding has ideal imperceptibility, and can effectively resist a variety of geometric transformation attacks. However, this technology only allows the watermarking data to be embedded in a single matrix, and is not suitable for multi-matrix, ignoring the human visual characteristics. Furthermore, according to formula (5), in order to prevent the gray value of the pixel from exceeding the boundaries of [0,255], the matrix B is simply added or subtracted. When the embedding parameter $\mathrm{k}$ is large, it is easy to cause greater distortion. In this paper, two boundary constraints are designed to improve the diamond coding technology by considering the human visual characteristics. This watermarking algorithm not only inherits the advantages of diamond coding technology, but also reduces the distortion of watermarking restoration.

\section{Hybrid Binary System}

In order to expand the capacity of watermarking, many scholars use hybrid binary system to implement [9-10]. Hybrid binary system mainly uses a series of cardinals and their corresponding numerical values to represent an integer [9]. For any integer q, it can be represented by different base numbers [9]:

$$
q=\left(g_{n-1}, g_{n-2}, \ldots g_{1}, g_{0}\right)_{\left\{b_{n-1}, \ldots b_{1}, b_{0}\right\}}
$$

Among them, $b_{i}$ is the base number; $g_{i} \in\left[0, b_{i}-1\right]$ is the value in Radix $b_{i}$, Given the matrix $\left\{b_{n-1}, . . b_{1}, b_{0}\right\}$, the number $g_{i}$ of $q$ in the matrix $b_{i}$ is:

$$
\begin{gathered}
g_{0}=\bmod \left(q, b_{0}\right) \\
g_{i}=\bmod \left(\frac{1}{\coprod_{j=0}^{i-} b_{j}}\left(q-g_{0}-\sum_{m=1}^{i-1}\left(g_{m} \times \prod_{j=0}^{m-1} b_{j}\right)\right), b_{i}\right)
\end{gathered}
$$

According to formula (6) and formula (9), integer q can be represented as:

$$
q=\sum_{m=1}^{i-1}\left(g_{i} \times \prod_{j=0}^{m-1} b_{j}\right)+g_{0}
$$

In order to describe the mixed binary system in detail, the decimal integer 314 is taken as an example, which can be represented as $314=(1,19,6)_{(13,25,7)}=1 \times 25 \times 7+19 \times 7+6$ or $314=(3,5,6)_{(7,13,7)}=3 \times 13 \times 7+5 \times 7+6$ with the help of 
radix. Therefore, for a given key data $S$, with the help of formula (6), it is changed into integer $q$; then, through formula (7) and formula (8), $q$ is changed into different cardinals and values.

\section{Pixel Interval Classification and Constraint Design}

In order to solve the deficiencies of diamond coding technology, this paper classifies the gray-scale intervals of the carrier image pixels to design boundary constraints and practice their adaptive adjustment. Firstly, the pixel gray value interval $[0,255]$ is divided into three non-overlapping sub-regions. Let $d_{i}$ be the gray value difference of a certain pair of pixels, $D\left(d_{i}\right)$ be the subinterval containing $d_{i}$, and $B\left(d_{i}\right)$ be the cardinal number corresponding to $d_{i}$ in $D\left(d_{i}\right) \cdot k_{l}, k_{h}$ is used to represent the two embedding parameters of upper and lower intervals in diamond coding. Similarly, if [0,255] is divided into three sub-intervals, the embedding parameters of upper, middle and lower intervals need to be expressed by threshold $T_{0}, T_{1}$ and $k_{l}, k_{m}, k_{n}$, as shown in Figure 2.

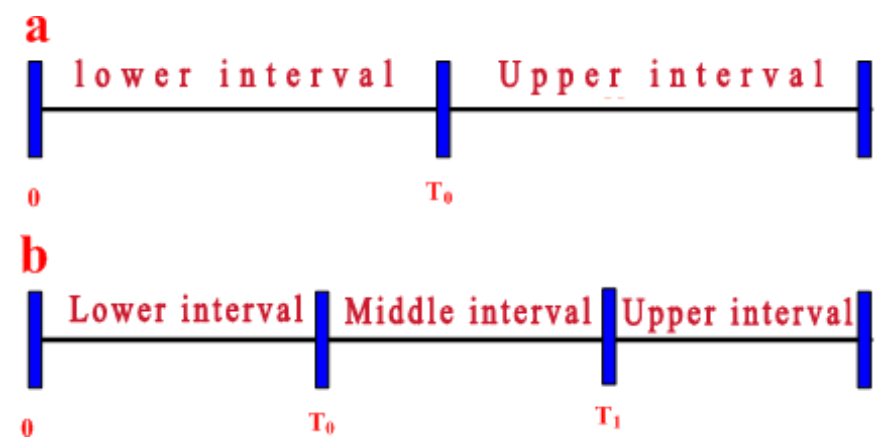

Fig.2:Interval segmentation of [0,255]. (a) two subintervals; (b) three subintervals.

In the lossless watermarking algorithm herein, the larger the pixel value difference $d_{i}$, the larger the base $B\left(d_{i}\right)$ corresponding to the embedded watermark data. If $d_{i}$ is within the lower interval $\left[0, T_{0}\right]$, the smaller parameter $k_{l}$ is accustomed to embed the watermark data into the pixel pair $\left(p_{i, 1}, p_{i, 2}\right)$ to reduce distortion. Conversely, if $d_{i}$ is located in the lower interval $\left[T_{0}, 255\right]$, the larger parameter $k_{n}$ is used to embed the watermark information to improve the embedding capacity. In short, the selection of the threshold $T_{0}, T_{1}$ and the embedded parameters $k_{l}, k_{m}, k_{n}$ depends primarily on the given watermark capacity. When the predetermined watermark capacity is small, the larger threshold is used, and the smaller base is used; otherwise, the smaller threshold is used, and the larger base is used.

According to the diamond coding technique, when the value $S_{B\left(d_{i}\right)}$ corresponding to the radix base $B\left(d_{i}\right)$ is inserted in the pixel pair $\left(p_{i, 1}, p_{i, 2}\right),\left(p_{i, 1}, p_{i, 2}\right)$ is revised to $\left(p_{i, 1}^{\prime}, p_{i, 2}^{\prime}\right)$. However, when the gray value of $p_{i, 1}^{\prime}, p_{i, 2}^{\prime}$ exceeds the interval $[0,255]$, there will be pixel overflow or underflow problems. In order to solve this problem, this paper needs to determine a $\left(p_{i, 1}, p_{i, 2}\right)$ and the closest pixel pair $\left(\hat{p}_{i, 1}, \hat{p}_{i, 2}\right)$ instead of $\left(p_{i, 1}, p_{i, 2}\right)$. In order to seek out the pixel pair $\left(\hat{p}_{i, 1}, \hat{p}_{i, 2}\right)$, this paper converts it to solve the optimal problem $(x, y)$ :

$$
\begin{gathered}
\text { minmize: } \quad\left(p_{i, 1}-x\right)^{2}+\left(p_{i, 2}-7\right)^{2} \\
\text { subject to : } \quad f(x, y)=S_{B\left(d_{i}\right)}, D\left(d_{i}\right)=D\left(\hat{d}_{i}\right) \\
0 \leq x, y \leq 255
\end{gathered}
$$

ISSN: 0010-8189 
$\hat{d}_{i}=|x-y|$.Equations (11) and (12) constitute a constraint to ensure that the characteristic value $D$ of $\left(\hat{p}_{i, 1}, \hat{p}_{i, 2}\right)$ is $S_{B\left(d_{i}\right)}$. Maintaining the same interval segmentation before and after the watermark data is embedded ensures that $\left(\hat{p}_{i, 1}, \hat{p}_{i, 2}\right)$ is within the interval $[0,255]$.

Since the value $S_{B\left(d_{i}\right)}$ is embedded in the pixel pair $\left(p_{i, 1}, p_{i, 2}\right)$, the interval $D\left(d_{i}\right)$ and $D\left(d_{i}^{\prime}\right)$ need to be consistent. If there is a difference between the two, there will be deviations in the restored watermark data. To this end, this paper defines a two pixel pair interval adaptive adjustment mechanism:

(10) $B\left(d_{i}\right)$ as the embedded base. In this case, the value $S_{B\left(d^{\prime}\right)}$ is in the base $B\left(d_{i}\right)$. According to the formula (10 equation (12), the pixel pair $\left(\hat{p}_{i, 1}, \hat{p}_{i, 2}\right)$ after embedding the watermark data can be obtained.

2Use $B\left(d_{i}^{\prime}\right)$ as the embedded base. The value $S_{B\left(d^{\prime}\right)}$ is in the base $B\left(d_{i}^{\prime}\right)$. According to the equations (10) to (12), the pixel pair $\left(\hat{p}_{i, 1}, \hat{p}_{i, 2}\right)$ after embedding watermark data can be obtained. However, you need to modify equation (11):

$$
f(x, y)=S_{B\left(d^{\prime}\right)}, D\left(\hat{d}_{i}\right)=D\left(d^{\prime}{ }_{i}\right)
$$

The above two pixel pair interval adaptive adjustment mechanism will lead to different embedding efficiency. For this reason, this paper selects a radix base $B(d)$ from $B\left(d_{i}^{\prime}\right), B\left(d_{i}\right)$ to provide greater watermark capacity and less distortion:

$$
d=\underset{d \in\left(d_{i}, d^{\prime}\right)}{\arg \max } \frac{\log _{2} B(d)}{\left(p_{i, 1}-\hat{p}_{i, 1}\right)^{2}+\left(p_{i, 2}-\hat{p}_{i, 2}\right)^{2}}
$$

\section{Lossless Watermarking Algorithm}

The lossless watermarking algorithm process of the mixed binary system and pixel difference is shown in Fig. 3 . It is mainly divided into two stages: (1) embedding of watermark data; (2) watermark information extraction. The specific steps are as follows:

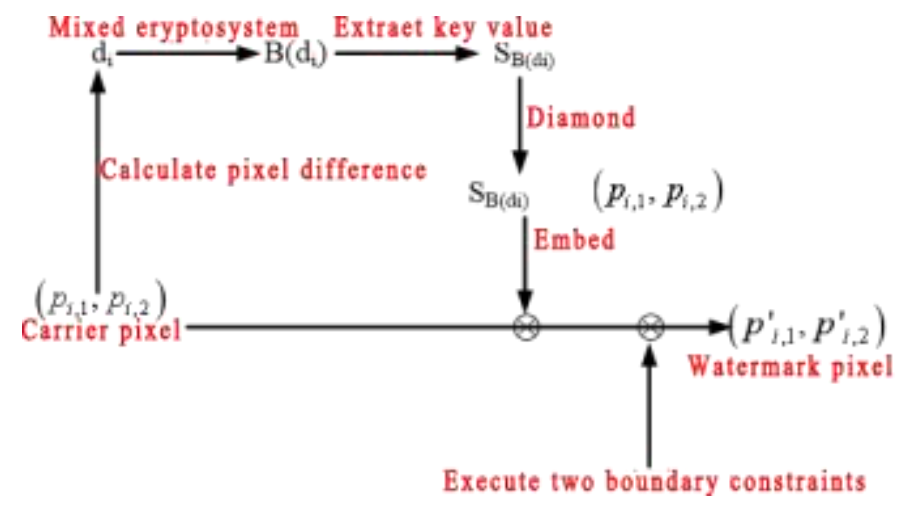

(a) Embedding of watermark data

Calculate pixel difference Extract embeded values

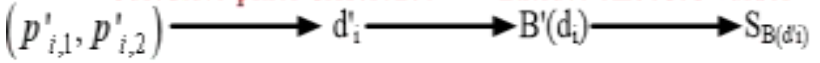

Watermark pixel Mixed cryptosystem Watermark data

(b) Extraction of watermark data

ISSN: 0010-8189

(C) CONVERTER 2020 
Fig.3: Watermarking Algorithm Process.

\section{1 embedding of watermark data}

1The size of the image $I$ is such that the watermark data to be inserted is $S$; at the same time, the pixel interval of the entire carrier image is divided into upper and lower subintervals by means of the threshold T0. And divide it into non-overlapping sub-blocks, and each sub-block is represented by a pixel pair $\left(p_{i, 1}, p_{i, 2}\right)$. Then, calculate the absolute pixel difference of each sub-block $d_{i}$ :

$$
d_{i}=p_{i, 1}-p_{i, 2}
$$

2Convert the watermark data s to an integer q according to equation (9).

3.According to the absolute difference $d_{i}$, define the cardinality corresponding to different characteristic pixels. $B_{i}$ Selection rule:

$$
B\left(d_{i}\right)= \begin{cases}B_{l} & d_{i} \leq T_{0} \\ B_{h} & d_{i}>T_{0}\end{cases}
$$

4Calculate $S_{B\left(d_{i}\right)}=\bmod \left(q, B\left(d_{i}\right)\right)$, embed $S_{B\left(d_{t}\right)}$ in the pixel pair $\left(p_{i, 1}, p_{i, 2}\right)$ according to the diamond encoding technique. Let the pixel pair after embedding the watermark data be $\left(p_{i, 1}^{\prime}, p_{i, 2}^{\prime}\right)$, then the absolute difference of the pixels is $d_{i}^{\prime}=p_{i, 1}^{\prime}-p_{i, 2}^{\prime}$.

5If $p_{i, 1}^{\prime}, p_{i, 2}^{\prime}$ is not in the interval $[0,255]$, there is an overflow or underflow problem. At this time, according to the solution process of the third subsection, the approximate pixel pair $\left(\hat{p}_{i, 1}, \hat{p}_{i, 2}\right)$ is found. Then, go to step 7.

6ff $D\left(d_{i}\right) \neq D\left(d_{i}^{\prime}\right)$ appears during the watermark insert process, the embedding base $B(d)$ and the relevant pixel pair $\left(\hat{p}_{i, 1}, \hat{p}_{i, 2}\right)$ are obtained according to equation (14).

7let $\left(p_{i, 1}^{\prime}, p_{i, 2}^{\prime}\right)=\left(\hat{p}_{i, 1}, \hat{p}_{i, 2}\right)$, and $q=\left(\frac{q-S_{B\left(d_{i}\right)}}{B\left(d_{i}\right)}\right)$.

8Repeat $1 \sim 7$ until $q=0$. At this time, the watermark image $I^{\prime}$ is output.

\subsection{Extraction of Watermark Data}

When the user obtains the watermark image $I^{\prime}$, and the corresponding threshold value t0 and the gray sub-interval parameters $k_{l}, k_{h}$ are also known, the process of extracting the watermark data from the pixel pair $\left(p_{i, 1}^{\prime}, p_{i, 2}^{\prime}\right)$ is exhibition in FIG. 3(b). The specific steps are as follows:

(1) The watermark image $I^{\prime}$ is divided into non-overlapping sub-blocks, each of which is represented by a pair of pixels $\left(p_{i, 1}^{\prime}, p_{i, 2}^{\prime}\right)$. Where $0 \leq i \leq M_{1} \times M_{2} / 2$. 
(2Calculate the pixel difference value $d_{i}^{\prime}=p_{i, 1}^{\prime}-p_{i, 2}^{\prime}$ of each sub-block according to equation (15). If $d_{i}^{\prime} \leq T_{0}$, the value corresponding to the embedded data is in the radix base $B\left(d_{i}^{\prime}\right)=2 k_{l}^{2}+2 k_{l}+1$; otherwise, the value is $B\left(d_{i}^{\prime}\right)=2 k_{h}^{2}+2 k_{h}+1$ in the radix base.

3Extracting values from $B\left(d_{i}^{\prime}\right)$ according to the diamond coding technique described in subsection 1;

4Repeat 1 3 until all inserted values have been extracted.

5Using equation (9), convert $S_{B\left(d^{\prime}\right)}$ to an int q by the hexadecimal technique corresponding to each value $B\left(d_{i}^{\prime}\right)$. Finally, the integer $q$ is converted to a binary representation to obtain the watermark data $S$.

\section{Simulation Results and Analysis}

In order to test the imperceptibility and capacity of the devised lossless watermarking technology, based on MB software, the watermark performance test was carried out, and the literature [5] and the literature [7] were used as the control group to reflect the superiority of the watermarking technique. The experimental conditions are: DELL, 3.5GHZ, dual-core CPU, 500GB hard drive and 8G memory. And in the USC-SIPI library [16], four 8-bit reference images are selected as the carrier image, as present in Fig. 4(a) to Fig.4(d), the sizes are all 256*256; using a pseudo random number generator to form The watermark data bit $S=111011_{2}$, and its corresponding decimal integer $q=59$. The other key parameters are: $T_{0}=30, k_{l}=1, k_{h}=2$. The technical evaluation technical indicators are: (1) imperceptibility and watermark capacity; (2) distortion.
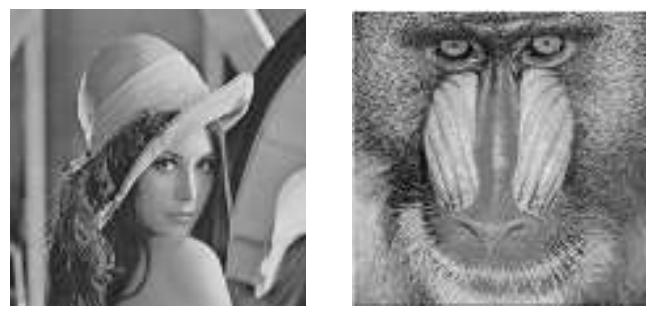

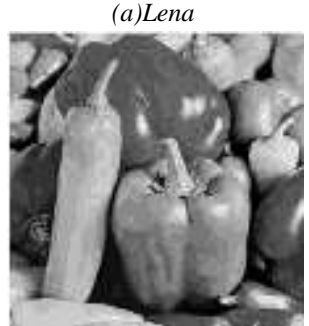

(c) Peppers (b) Mandrill

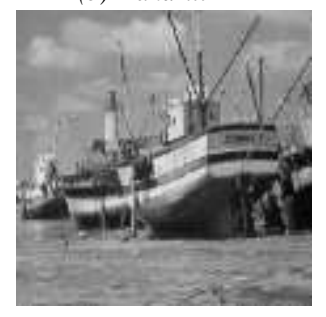

(d) Boat

Fig.4:Vector image used in the experiment.

6.1 Availability of Data and Materials

The Fig. 4(a) to Fig. 4(d) supporting the findings of the article is available in the [The USC-SIPI Image Database] at [http://sipi.usc.edu/database/database.php?volume=misc], reference number [4.1.04, 4.2.03, 4.2.07, boat.512].

\subsection{Unperceived Performance and Watermark Capacity Test Analysis}

Impossibility and watermark capacity are the most commonly used measurement indicators for watermarking technology, and they are also important evaluation methods that reflect the security and practicability of its ISSN: 0010-8189

(C) CONVERTER 2020 
algorithm [11-12]. For this reason, based on the algorithm of this paper, the literature [7] and the literature [5], the embedding rate is set to $0.8 \mathrm{bpp}$, and the watermark data $S=111011_{2}$ is embedded in the carrier image. The obtained watermark image is exhibition in Figure 5 7. According to the watermark results, the three watermarking techniques have good imperceptibility. The watermark data is fully hidden into the carrier image, and there is no visual leakage of information. The output image is almost the same as the initial carrier. By calculating the visual similarity between the watermark image of the three algorithms and the initial carrier, the watermark image of the three algorithms has a pixel degree of 0.99 , which is very close to 1 . Further quantify the difference between the imperceptibility of the three watermarking techniques, this paper tests the SSIM values between the watermark image obtained by the three algorithms at different embedding rates and the initial image. The obtained data are exhibition in Table 1. It can be seen from the table that the SSIM values of the watermark images of the two techniques of literature [5] and [7] are slightly higher than the algorithm of this paper, but the degree of difference is small, which shows that the algorithm is equivalent to the literature [5] and [7]. Level imperceptibility. The reason is that the literature [5] and the literature [7] encrypt the watermark information before embedding it into the carrier image, and confuse its pixels, so that the imperceptibility of the two is further improved, which is slightly higher than the proposed algorithm. The algorithm in this paper directly embeds the watermark information into the carrier. Although it is not encrypted, the algorithm considers the human visual characteristics and embeds more watermark capacity into pixel pairs with larger pixel differences. These pixels are not attractive to the eye, making the proposed technology equally ideally imperceptible.

In addition, according to Table 1, when the embedding rate surpass $2.4 \mathrm{bpp}$, the value of the watermark image can still be maintained at $39.065 \mathrm{~dB}$, while the two techniques of literature [5] and [7] fail to embed the watermark, which shows the proposed algorithm. Watermarking technology has a large watermark capacity. The main reason is that the watermarking technique adaptively adjusts the watermark data embedding capacity of each pixel according to the pixel difference value and its corresponding mixed radix base. For the pixel pair with larger difference, the larger radix base is selected. Pixel interval constraints, using the improved diamond coding technique, embed larger data capacity, and embed small-capacity watermark data into the pixels of the radix base, which fully improves the watermark capacity of the algorithm; and the literature [5] The literature [7] ignores the pixel characteristics of the carrier image and embeds the same amount of data into each pixel, making its watermark capacity low.

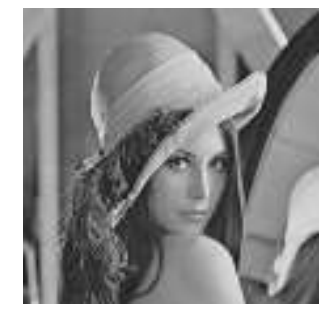

(a) Lena with water-printed $\mathrm{S}$ (b) $\mathrm{SSIM}=0.9992$

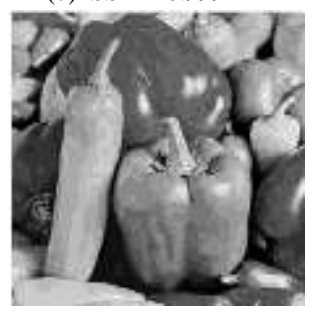

(c) Peppers with watermark S SSIM=0.9991

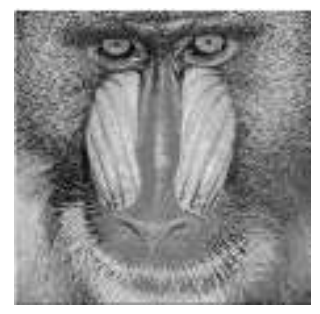

(b) Mandrill with watermark $\mathrm{S}$ SSIM $=0.9989$

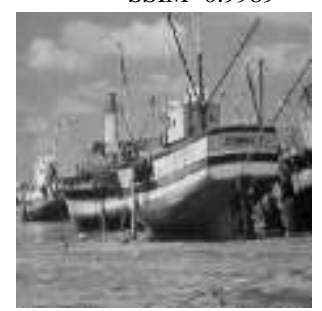

(d) Boat with watermark SSIM=0.9993

Fig.5: Watermark embedding results of the algorithm in this paper

ISSN: 0010-8189 


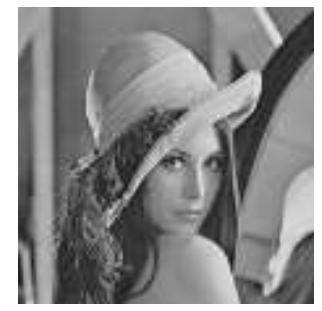

(a) Lena with water-printed $\mathrm{S}$ SSIM $=0.9994$

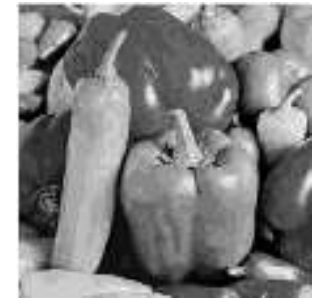

(c) Peppers with watermark $\mathrm{S}$ SSIM=0.9993

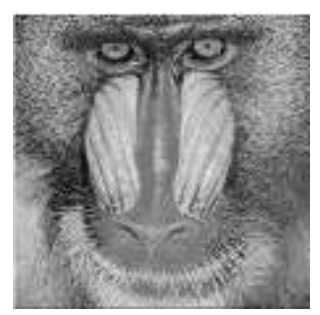

(b) Mandrill with watermark S SSIM $=0.9991$

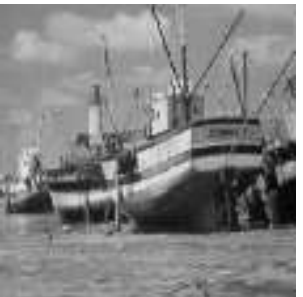

(d) Boat with watermark $\mathrm{S}$ $\mathrm{SSIM}=0.9996$

Fig.6: Watermark embedding results in [5]

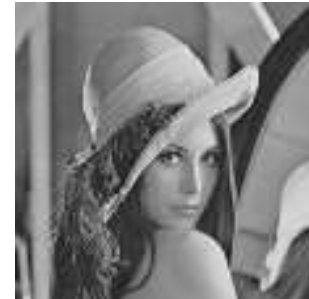

(a) Lena with water-printed $\mathrm{S}$

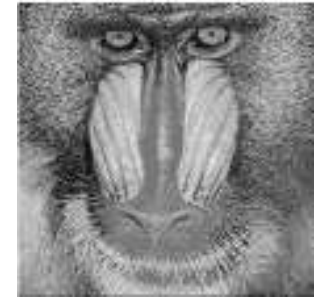

SSSIM $=0.9998$

b) Mandrill with water-stained S

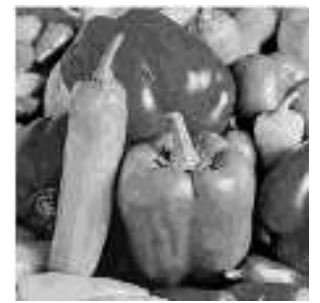
SSIM $=0.9993$

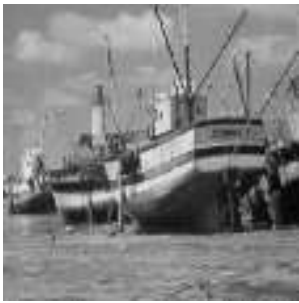

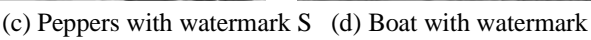
$\mathrm{SSIM}=0.9995$ SSIM $=0.9997$

Fig.7: Watermark embedding results in [7]

Table 1 Capacity and Imperceptibility Testing of Different Watermarking Algorithms

\begin{tabular}{|c|c|c|c|c|}
\hline $\begin{array}{l}\text { Embedding } \\
\text { rate(bpp) }\end{array}$ & $\begin{array}{l}\text { Capacity } \\
\text { (bits) }\end{array}$ & $\begin{array}{c}\text { Carrier } \\
\text { image }\end{array}$ & $\begin{array}{l}\text { Watermarking } \\
\text { algorithm }\end{array}$ & $\begin{array}{l}\text { PSNR }(\mathrm{dB}) \text { of the } \\
\text { watermark image }(\mathrm{dB})\end{array}$ \\
\hline \multirow{3}{*}{0.8} & \multirow{3}{*}{387900} & \multirow{3}{*}{ Lena } & Algorithm & 46.59 \\
\hline & & & Literature[5] & 48.36 \\
\hline & & & Literature[7] & 49.02 \\
\hline \multirow{3}{*}{1.6} & \multirow{3}{*}{748217} & \multirow{3}{*}{ Mandrill } & Algorithm & 44.75 \\
\hline & & & Literature[5] & 42.28 \\
\hline & & & Literature[7] & 42.95 \\
\hline \multirow{3}{*}{2.4} & \multirow{3}{*}{891750} & \multirow{3}{*}{ Peppers } & Algorithm & 41.73 \\
\hline & & & Literature[5] & I \\
\hline & & & Literature[7] & I \\
\hline
\end{tabular}

ISSN: 0010-8189

(C) CONVERTER 2020 


\begin{tabular}{|l|l|l|l|l|}
\hline \multirow{3}{*}{3.2} & \multirow{4}{*}{ Boat } & Algorithm & 39.06 \\
\cline { 3 - 4 } & \multirow{3}{*}{1061230} & Literature[5] & $/$ \\
\cline { 3 - 4 } & & Literature[7] & $/$ \\
\hline
\end{tabular}

\subsection{Algorithm for Distortion Test}

In addition to imperceptibility and algorithm capacity, the anti-falsification of the algorithm is also a key index to evaluate the watermarking technology. The better the anti-distortion performance is, the higher the quality of the extracted watermark and carrier image will be [13-14]. To this end, this paper takes the Lena image as the target, and uses the watermark extraction mechanism of the three algorithms to recover the watermark data and the carrier image from Fig. 5(a), Fig. 6(a) and Fig. 7(a), according to the literature [15]. The method quantifies the distortion by testing the difference histograms of the three, and the results are shown in Fig.8. It can be seen from the figure that the frequency distribution of the restored carrier image is the closest to that of the original carrier image, and the deviation between them is very low. However, the two techniques of literature [5] and [7] have poor antidistortion performance, and the restoration carrier The distortion of the image is higher than the proposed technique, and the frequency domain distribution of the restored carrier image has a large deviation from the initial carrier image, especially in the literature [7]. In addition, in order to quantify the distortion of the restored watermark information corresponding to the three algorithms, this paper extracts the watermark data from the watermark images of the respective algorithms in Figure 5 7, and tests the three algorithms under different embedding rates. [5], PSNR curve of the restored watermark data in [7], See Figure 9 for the results. According to the test data, when the embedding rate of watermark data increases gradually, the PSNR values of the restored watermark data of the three algorithms show a downward trend. However, compared with literature [5] and literature [7], the PSNR value of the watermark technology in this paper is always the largest, and the maximum information embedding capacity of each pixel in the carrier image can reach 3.2bpp; The PSNR values of references [5] and [7] are smaller than those of the proposed technique, and the maximum embedding rate of each pixel is 2.0 BPP. This shows that the proposed algorithm not only has larger watermark capacity, but also has the highest accuracy and the lowest distortion. The main reason is that with the help of threshold, the proposed watermarking technology divides the gray range of pixels into two continuous sub ranges, and combines the pixel difference to design two constraint conditions of the pixel range, which effectively solves the overflow and underflow of the pixel value and minimizes the distortion of the restored carrier image; However, in the process of embedding watermark data in literature [5] and [7], the overflow and underflow of pixel value are ignored, which leads to a large degree of distortion.

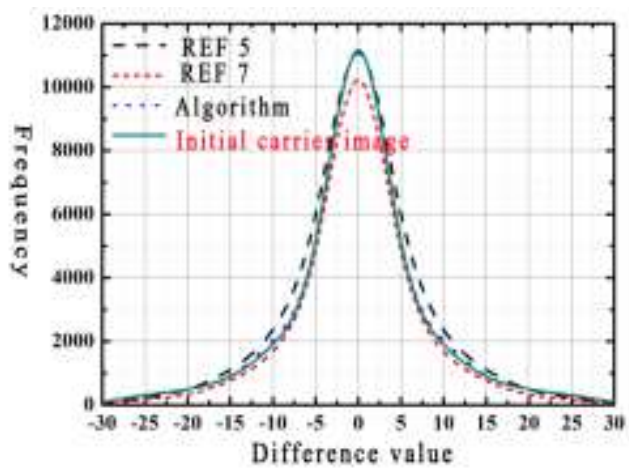

Fig.8: Three Algorithms to Restore the Difference Histogram Test Results of the Carrier Image

ISSN: 0010-8189 


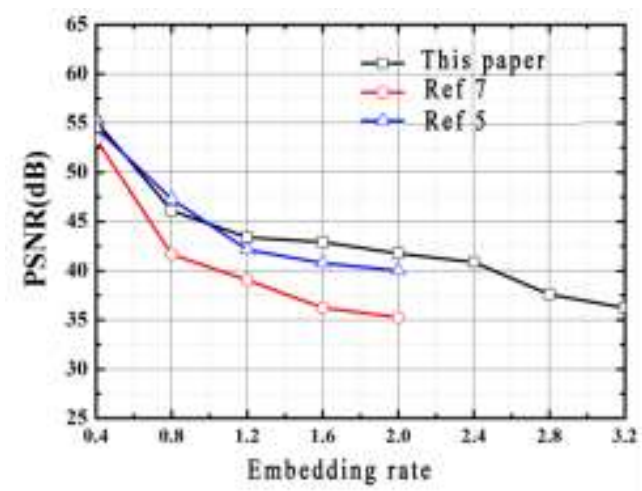

(a) Lena image

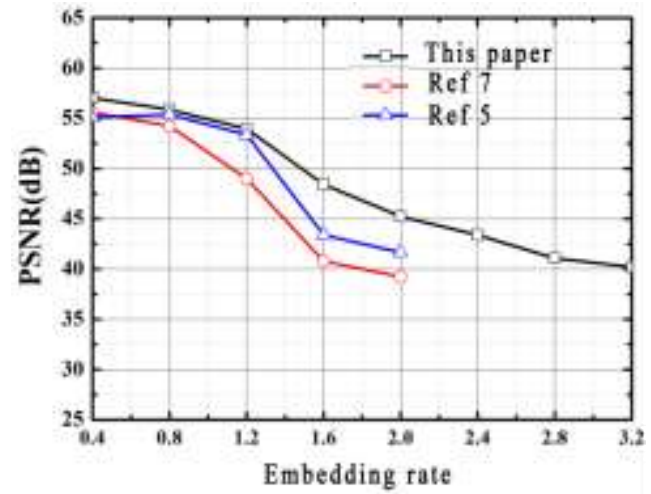

(b) Mandrill image

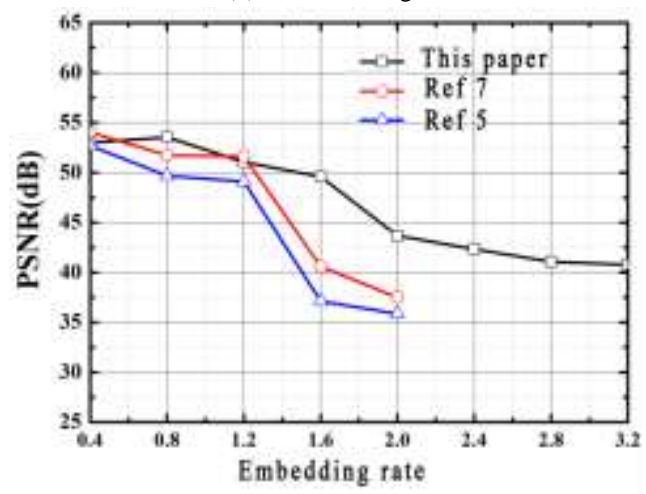

(c) Peppers image

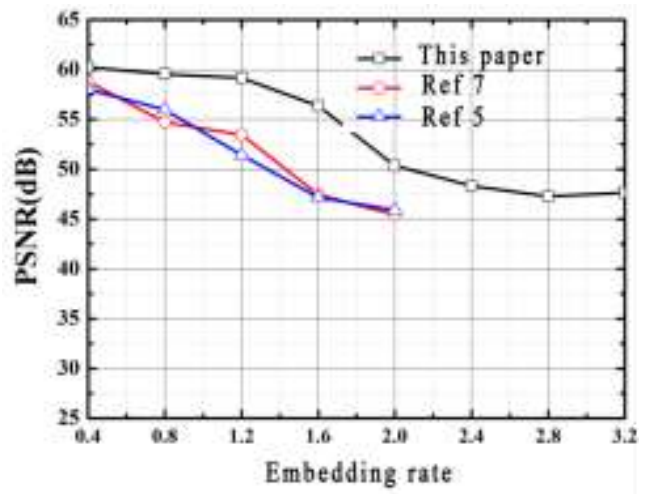

(d) Boat

Fig.9: Watermark Image Restoration Quality of Different Algorithms

\section{CONCLUSION}

ISSN: 0010-8189

(c) CONVERTER 2020

www.converter-magazine.info 
To reduce the distortion of the watermarking algorithm and improve the watermarking capacity, this paper designs a data watermarking algorithm based on pixel difference and the hybrid system. The algorithm adaptively embeds watermark data of different capacities into each pixel based on the difference of the sub-block pixel pairs. The carrier image is segmented into sub-blocks, and the pixel difference value of the sub-image block is calculated; and based on the mixed cryptosystem, a plurality of radix base numbers corresponding to the watermark data are obtained. Considering the visual characteristics of human eyes, design the adaptive adjustment mechanism of pixel interval, improve the diamond coding technology, realize the adaptive embedding of watermark information, embed more watermark data into pixel pairs with larger difference, and hide a small amount of data. In the subblock with smaller difference, the watermark capacity is maximized and the watermark quality is improved. At the same time, the watermark information extraction mechanism is designed to extract the watermark data. The experimental data verified the rationality and superiority of the proposed watermarking technology.

\section{Current and Future Developments}

Lossless digital watermarking is also called reversible digital watermarking. It is to embed the watermark into the multimedia data under the premise of not affecting the usability of the multimedia. When the content is suspected, the watermark can be proposed for the authenticity of the multimedia content. The current research is mainly based on typical lossless invisible and visible digital watermarking algorithms in the airspace, wavelet domain, etc.

Future research directions are mainly divided into the following three directions: Research on unified attack model and evaluation system of lossless watermarking algorithm; Research on multilevel watermarking technology; Research on watermark extraction method in lossless watermark algorithm.

\section{Acknowledgements}

This work is supported by National Natural Science Foundation of China (71371172): Research on Enterprise Resource Placement Optimization Based on Internet of Things, Big data copyright protection system based on human biological characteristics and its application.

\section{References}

[1] Qingtang Su, Gang Wang, Xiaofeng Zhang. An improved color image watermarking algorithm based on QR decomposition, Multimedia Tools and Applications, vol.76, no.1: 707-729, 2017.

[2] A high capacity reversible data hiding scheme based on right-left shift. Weiqing Wang, Junyong Ye,Tongqing Wang,Weifu Wang. Signal Processing. 2018.

[3] Image steganography in spatial domain: A survey. Mehdi Hussain, Ainuddin Wahid Abdul Wahab,Yamani Idna Bin Idris,Anthony T.S. Ho, Ki-Hyun Jung. Signal Processing: Image Communication. 2018.

[4] Dandan Zhu, Lizhi Lv. A New Image Watermarking Algorithm Using the Contourlet Transform and the Harris Detector, Computer Vision, vol.547, no.33:439-477, 2015.

[5] Zhengwei Zhang, Lifa Wu, Shaozhang Xiao. Adaptive reversible image watermarking algorithm based on IWT and level set, EURASIP Journal on Advances in Signal Processing, vol.5, no.1:10-18, 2017.

[6] Xiang-yang Wang; Si-yu Zhang; Tao-tao Wen. Coefficient difference based watermark detector in nonsubsampled contourlet transform domain .Information Sciences, vol.176, pp. 274-290, 2019.

[7] ChunHung Chen, Yuan-Liang Tang, Chih-Peng Wang. A robust watermarking algorithm based on salient image features, Optik - International Journal for Light and Electronic, vol.125, no.3:1134-1140, 2014.

[8] Reversible data hiding using multi-pass pixel value ordering and prediction-error expansion. Wenguang He,Ke Zhou,Jie Cai,Long Wang,Gangqiang Xiong. Journal of Visual Communication and Image Represe. 2017

[9] Optimal PPVO-based reversible data hiding. Shaowei Weng, Guohao Zhang, Jeng-Shyang Pan,Zhili Zhou. Journal of Visual Communication and Image Represe. 2017

[10] Liu Jiuwen, Pan Feng, Li Jun. A large capacity steganographic algorithm combined with error image ISSN: 0010-8189

(C) CONVERTER 2020

www.converter-magazine.info 
and modular operation, small micro computer system, vol.36, no.8:1882-1885, 2015.

[11] Dawen Xua, Rangding Wang. An improved scheme for data hiding in encrypted H.264/AVC videos, Journal of Visual Communication and Image Representation, vol.36, no.7:229-242, 2016.

[12] Zhao Bo, Qin Guihe. Image watermarking algorithm with high robustness, Journal of Jilin University (Engineering Edition), vol.47, no.1:249-254, 2016.

[13] Li Shuya, Hu Qin, Deng Xiaohong. Gray level co-occurrence matrix, texture feature selection, reversible image watermarking, Journal of photoelectron, laser, vol.5, no.4:411-418, 2017.

[14] KaCheng Choi, ChiMan Pun. Robust lossless digital watermarking using integer transform with Bit plane manipulation, 2016, Multimedia Tools and Applications, vol.75, no.11:1-25, 2016.

[15] Li Shuya, Hu Qin, Deng Xiaohong. Reversible image watermarking with omnidirectional gradient prediction and adaptive block selection small and micro computer system, vol.38, non.1:174-178, 2017.

[16] H Zhang, Q Guan, X Zhao, Steganography Based on Adaptive Pixel-Value Differencing Scheme Revisited, International Workshop on Digital Watermarking, vol.12, no.8:32-47, 2013. 